\title{
The Number of Linear Extensions of the Boolean Lattice
}

\author{
Graham R. Brightwell * \\ Department of Mathematics \\ London School of Economics \\ Houghton St. \\ London WC2A 2AE \\ England \\ Prasad Tetali ${ }^{\dagger}$
}

School of Mathematics \& College of Computing

Georgia Tech.

Atlanta

GA 30332-0160

U.S.A.

28 October 2003

CDAM Research Report LSE-CDAM-2003-19

\begin{abstract}
Let $L\left(Q^{t}\right)$ denote the number of linear extensions of the $t$-dimensional Boolean lattice $Q^{t}$. We use the entropy method of Kahn to show that

$$
\frac{\log \left(L\left(Q^{t}\right)\right)}{2^{t}}=\log \left(\begin{array}{c}
t \\
\lfloor t / 2\rfloor
\end{array}\right)-\frac{3}{2} \log e+o(1),
$$

where the logarithms are base 2 . We also find the exact maximum number of linear extensions of a $d$-regular bipartite order on $n$ elements, in the case when $n$ is a multiple of $2 d$.
\end{abstract}

*Email: g.r.brightwell@lse.ac.uk

${ }^{\dagger}$ Research supported in part by NSF grant DMS-0100289; email: tetali@math.gatech.edu 


\section{Introduction}

Let $L(P)$ denote the number of linear extensions of a poset $P$. A natural problem is to estimate $L(P)$ when $P$ is the Boolean lattice $Q^{t}$, consisting of the subsets of $[t]=$ $\{1,2, \ldots, t\}$, ordered by inclusion. This problem was apparently first posed by Richard Stanley (see Sha and Kleitman [14]), although it has also been raised by several others independently.

The Boolean lattice $Q^{t}$ is naturally partitioned into its level sets (or simply levels) $A_{j}$, where $A_{j}$ consists of those subsets of $[t]$ of size exactly $j$. A trivial lower bound on $L\left(Q^{t}\right)$ is $\prod_{j=0}^{t}\left(\begin{array}{c}t \\ j\end{array}\right)$ !, since this counts exactly those linear extensions of $Q^{t}$ in which all of the $j$ th level comes below the $(j+1)$ st, for each $j$.

A trivial upper bound is $\left(\begin{array}{c}t \\ \lfloor t / 2\rfloor\end{array}\right)^{2^{t}}$; to see this, consider writing down a linear extension from the bottom up - at each stage the set of possible next elements is an antichain in $Q^{t}$, and so has size at most $\left(\begin{array}{c}t \\ \lfloor t / 2\rfloor\end{array}\right)$. For many purposes, these bounds are sufficiently close: they can be written as

$$
\log \left(\begin{array}{c}
t \\
\lfloor t / 2\rfloor
\end{array}\right)-\frac{3}{2} \log e+o(1) \leq \frac{\log \left(L\left(Q^{t}\right)\right)}{2^{t}} \leq \log \left(\begin{array}{c}
t \\
\lfloor t / 2\rfloor
\end{array}\right) .
$$

Here and throughout, log denotes the base 2 logarithm.

The only previous improvement on these trivial bounds was made by Sha and Kleitman [14], who improved the upper bound to

$$
L\left(Q^{t}\right) \leq \prod_{j=0}^{t}\left(\begin{array}{l}
t \\
j
\end{array}\right)^{\left(\begin{array}{l}
t \\
j
\end{array}\right)} \leq \prod_{j=0}^{t}\left(\begin{array}{l}
t \\
j
\end{array}\right) ! \exp \left(2^{t}\right),
$$

yielding

$$
\frac{\log \left(L\left(Q^{t}\right)\right)}{2^{t}} \leq \log \left(\begin{array}{c}
t \\
\lfloor t / 2\rfloor
\end{array}\right)-\frac{1}{2} \log e+o(1)
$$

Sha and Kleitman suggested that the simple lower bound in fact gives the right value of the constant term in the asymptotic expansion for $\log \left(L\left(Q^{t}\right)\right) / 2^{t}$, namely $\frac{3}{2} \log e$. They also remarked that the lower bound can be improved slightly to

$$
\frac{\log L\left(Q^{t}\right)}{2^{t}} \geq \log \left(\begin{array}{c}
t \\
\lfloor t / 2\rfloor
\end{array}\right)-\frac{3}{2} \log e+\frac{C}{t}
$$

for some positive constant $C$. Our primary purpose in this paper is to prove the following result, which indeed shows that the constant term in the asymptotic expansion is $\frac{3}{2} \log e$, and gets reasonably close to the error term.

Theorem 1.1 For any positive integer $t$,

$$
L\left(Q^{t}\right) \leq \exp \left(2^{t} \frac{6 \ln t}{t}\right) \prod_{j=0}^{t}\left(\begin{array}{l}
t \\
j
\end{array}\right) ! .
$$




\section{Corollary 1.2}

$$
\frac{\log \left(L\left(Q^{t}\right)\right)}{2^{t}}=\log \left(\begin{array}{c}
t \\
\lfloor/ 2\rfloor
\end{array}\right)-\frac{3}{2} \log e+O\left(\frac{\ln t}{t}\right)
$$

Theorem 1.1 supports the intuition that, in a typical linear extension of $Q^{t}$, most of the $j$ th level appears below most of the $(j+1)$ st, for each $j$.

Theorem 1.1 is a special case of a more general result. A ranked poset $P$ is a poset in which every maximal chain has the same length. A ranked poset is naturally partitioned into ranks $A_{1}, \ldots, A_{k}$, where $A_{j}$ contains those elements $x$ such that the longest chain with top element $x$ has exactly $j$ elements. In a ranked poset, if $x \in A_{j}$ is covered by an element $y$, then $y \in A_{j+1}$. Also, every element in $A_{j}$ is above some element of $A_{j-1}$ (provided $j>1$ ) and below some element of $A_{j+1}$ (provided $j<k$ ).

For a positive integer $k \geq 2$, and for $j=1,2, \ldots, k$, let $n_{j}, u_{j}$, and $d_{j}$ be arbitrary positive integers. Now let $P$ be an $n$-element ranked poset with ranks $A_{1}, A_{2}, \ldots, A_{k}$, of sizes $n_{1}, n_{2}, \ldots, n_{k}$ respectively, such that every element of $A_{j}$ is above exactly $d_{j}$ elements of $A_{j-1}(j \geq 2)$, and every element of $A_{j}$ is below exactly $u_{j}$ elements of $A_{j+1}$ $(j \leq k-1)$. Finally let $r=\sum_{j=2}^{k}\left(n_{j-1} / d_{j}\right)$.

Theorem 1.3 For $P$ a poset as above, the number $L(P)$ of linear extensions of $P$ satisfies

$$
L(P) \leq\left(\frac{2 e(k-1) n}{r}\right)^{r} n_{1} ! \cdots n_{k} ! .
$$

Of course, $n_{1} ! \cdots n_{k}$ ! is a lower bound on $L(P)$ for such a poset $P$. We get a useful bound whenever $r=o(n / \ln k)$; if we think of $r$ as approximately $n / \tilde{d}$, where $\tilde{d}$ is the harmonic mean of the "down-degrees" of elements of $P$, then this corresponds to the mild condition $\tilde{d}=\omega(\ln k)$. In this case, the multiplicative "error term" $\left(\frac{2 e(k-1) n}{r}\right)^{r}$ is $2^{o(n)}$; in the context of counting linear extensions, this means that $n_{1} ! \cdots n_{k}$ ! is a good estimate of $L(P)$.

Our proof of this theorem is based on what seems to be emerging as an "entropy method," stemming from Kahn's proof [10] of a result of Kleitman and Markowsky on Dedekind's problem concerning the number of antichains in the Boolean lattice. Other applications of this method can be found in [2], [8] and [9].

In the case where there are just two ranks, i.e., the poset $P$ is bipartite, we are able to prove a more precise result.

Theorem 1.4 Let $P$ be a ranked poset on $n$ elements, with two ranks $A$ and $B$, such that every element of $A$ is below exactly $u$ elements of $B$, and every element of $B$ is above exactly d elements of $A$. Then

$$
L(P) \leq n !\left(\begin{array}{c}
d+u \\
u
\end{array}\right)^{-n /(d+u)} .
$$


This result is best possible for $n$ a multiple of $d+u$. Indeed the expression given is the number of linear extensions of the disjoint union of $n /(d+u)$ copies of the complete bipartite poset $K_{d, u}$ with $d$ minimal and $u$ maximal elements.

Perhaps curiously, we cannot replace "exactly" by "at least" in the degree conditions of Theorem 1.4. Indeed, for $n$ large, form a bipartite poset $P$ with some $d$ elements of $A$ below all elements of $B$, some $u$ elements of $B$ above all elements of $A$, and no other relations. Then $L(P) \geq(n-d-u)$ !, which is much larger than the bound in Theorem 1.4.

Fishburn and Trotter [7] studied a seemingly related problem, where they fixed the number $n$ of elements and the number $m$ of comparable pairs, and sought to maximize the number of linear extensions. They proved that the extremal poset for their problem is always a semiorder; our extremal examples are not.

In Section 2, we provide the necessary background on entropy. Then we prove Theorem 1.4 in Section 3; this proof is particularly close to one given by Kahn in [9].

In Section 4, we give the proof of Theorem 1.3, which requires overcoming various technical difficulties. Theorem 1.1 follows almost immediately from Theorem 1.3.

The result, and the method, of Sha and Kleitman was extended by Shastri [15] to cover any ranked poset satisfying the LYM condition, the sizes of whose ranks form a symmetric unimodal sequence. In Section 5, we show that the Sha-Kleitman bound $L(P) \leq \prod_{j=1}^{k} n_{j}^{n_{j}}$ holds for any ranked poset satisfying the LYM condition, regardless of the degrees and rank sizes $n_{1}, \ldots, n_{k}$ : in fact this is an immediate consequence of a result of Kahn and Kim [11] - for which we supply an alternative proof that might be of some interest.

\section{Basics on Entropy}

In this section we recall various basic facts on entropy, including a powerful lemma of Shearer. Our presentation follows that in [9] very closely. Some standard references for material on entropy include [5], [6], and [12].

Let $\mathbf{X}, \mathbf{Y}$, and $\mathbf{Z}$ denote arbitrary discrete random variables. The entropy of $\mathbf{X}$ is defined to be

$$
H(\mathbf{X})=\sum_{x} p(x) \log \frac{1}{p(x)}
$$

where $p(x)=\mathbf{P}(\mathbf{X}=x)$. The conditional entropy of $\mathbf{X}$ given $\mathbf{Y}$ is

$$
H(\mathbf{X} \mid \mathbf{Y})=\mathbf{E} H(\mathbf{X} \mid \mathbf{Y}=y)=\sum_{y} p(y) \sum_{x} p(x \mid y) \log \frac{1}{p(x \mid y)},
$$

where $p(x \mid y)=\mathbf{P}(\mathbf{X}=x \mid \mathbf{Y}=y)$.

For a random vector $\mathbf{X}=\left(\mathbf{X}_{1}, \ldots, \mathbf{X}_{n}\right)$, viewed as a random variable, the chain rule of entropy tells us that

$$
H(\mathbf{X})=H\left(\mathbf{X}_{1}\right)+H\left(\mathbf{X}_{2} \mid \mathbf{X}_{1}\right)+\cdots+H\left(\mathbf{X}_{n} \mid \mathbf{X}_{1}, \ldots, \mathbf{X}_{n-1}\right) .
$$


We will also make use of the inequalities

$$
\begin{gathered}
H(\mathbf{X}) \leq \log |\operatorname{range}(\mathbf{X})|, \quad \text { with equality if } \mathbf{X} \text { is uniform, } \\
H(\mathbf{X} \mid \mathbf{Y}) \leq H(\mathbf{X})
\end{gathered}
$$

and more generally,

$$
\text { if } \mathbf{Y} \text { determines } \mathbf{Z} \text { then } H(\mathbf{X} \mid \mathbf{Y}) \leq H(\mathbf{X} \mid \mathbf{Z}) \text {, }
$$

where "Y determines $\mathbf{Z}$ " means formally that $H(\mathbf{Z} \mid \mathbf{Y})=0$. Note that (2) and (4) imply

$$
H(\mathbf{X}) \leq H(\mathbf{Y})+H(\mathbf{X} \mid \mathbf{Y})
$$

and also the sub-additive property of entropy:

$$
H\left(\mathbf{X}_{1}, \ldots, \mathbf{X}_{n}\right) \leq \sum H\left(\mathbf{X}_{i}\right)
$$

We also have a conditional version of (6):

$$
H\left(\mathbf{X}_{1}, \ldots, \mathbf{X}_{n} \mid \mathbf{Y}\right) \leq \sum H\left(\mathbf{X}_{i} \mid \mathbf{Y}\right)
$$

Finally we need the following lemma of Shearer (see page 33 in [4]), which refines the sub-additivity property (6). A particularly elegant and short proof of this useful lemma is given in [13]. For a random vector $\mathbf{X}=\left(\mathbf{X}_{1}, \ldots, \mathbf{X}_{m}\right)$ and $A \subseteq[m]$, set $\mathbf{X}_{A}=\left(\mathbf{X}_{i}: i \in A\right)$.

Lemma 2.1 Let $\mathbf{X}=\left(\mathbf{X}_{1}, \ldots, \mathbf{X}_{m}\right)$ be a random vector and $\mathcal{A}$ a collection of (not necessarily distinct) subsets of $[m]$, with each element of $[m]$ contained in at least d members of $\mathcal{A}$. Then

$$
H(\mathbf{X}) \leq \frac{1}{d} \sum_{A \in \mathcal{A}} H\left(\mathbf{X}_{A}\right)
$$

The conditional analog of Shearer's lemma, where all entropies are taken conditional on the same random variable $\mathbf{Y}$, is true as well, and we make use of it in our proof.

\section{Bipartite posets}

In this section, we prove Theorem 1.4.

Let $C_{M}$ denote the $M$-element chain with groundset $[M]=\{1, \ldots, M\}$, with the standard order. We make use of an idea of Shepp [16, 17]: rather than work directly with linear extensions, we count the number $O_{M}(P)$ of order-preserving maps from the $n$-element poset $P$ to $C_{M}$, i.e., functions $f$ from $P$ to $C_{M}$ such that $x<y$ implies $f(x) \leq f(y)$. The connection with linear extensions is given by the following easy lemma, first observed by Shepp. 
Lemma 3.1 For any $n$-element poset $P$,

$$
\lim _{M \rightarrow \infty} \frac{O_{M}(P)}{M^{n}}=\frac{L(P)}{n !}
$$

Thus Theorem 1.4 will be an easy consequence of the following result.

Theorem 3.2 Let $P$ be an n-element ranked poset with two ranks $A$ and $B$, such that each element of $A$ is below exactly $u$ elements of $B$, and each element of $B$ is above exactly $d$ elements of $A$, and let $M$ be any natural number. Then

$$
O_{M}(P) \leq\left(\sum_{j=1}^{M}\left[(M-j+1)^{u}-(M-j)^{u}\right] j^{d}\right)^{n /(d+u)}=O_{M}\left(K_{d, u}\right)^{n /(d+u)} .
$$

To see the final identity, note that the $j$-term in the sum counts order-preserving maps $f$ of $K_{d, u}$ in which $\min _{y \in B} f(y)$ is equal to $j$. Of course, here too we have equality when $P$ is the disjoint union of copies of $K_{d, u}$.

For $M=2$, order-preserving maps from $P$ to $C_{2}$ are in 1-1 correspondence with antichains in $P$, which in turn are exactly independent sets in the (bipartite) comparability graph. So the number of independent sets in a " $(u, d)$-biregular" bipartite graph on $n$ vertices is at most $\left(2^{u}+2^{d}-1\right)^{n /(d+u)}$, with equality if the graph is a disjoint union of $K_{d, u} \mathrm{~s}$. This result is in fact Theorem 4.3 of Kahn [9], and in this case our proof is nothing more than a direct translation of Kahn's. Indeed, our proof of Theorem 3.2 follows Kahn's proof very closely.

Proof. Let $P$ be a poset with ranks $A$ and $B$ satisfying the conditions of the theorem, and let $\mathbf{f}$ be a uniform random variable over all elements of $O_{M}(P)$. Set $a=|A|$ and $b=|B|$, so that $n=a+b$.

For $X$ a subset of the ground-set of $P$, let $\mathbf{f}_{X}$ be the restriction of $\mathbf{f}$ to $X$. For $x \in A$, let $U(x)$ be the set of elements in $B$ covering $x$, and define the random variable $\mathbf{Y}_{x}=\min \mathbf{f}_{U(x)}$. Finally, for $x \in A$ and $j \in[M]$, let $p_{x}(j)$ be the probability that $\mathbf{Y}_{x}$ is equal to $j$.

Now we have

$$
\log O_{M}(P)=H(\mathbf{f})=H\left(\mathbf{f}_{B}\right)+H\left(\mathbf{f}_{A} \mid \mathbf{f}_{B}\right) .
$$

If $B=\left\{y_{1}, \ldots, y_{b}\right\}$, set $\mathbf{f}_{i}=\mathbf{f}\left(y_{i}\right)$, and think of $\mathbf{f}_{B}$ as the vector $\left(\mathbf{f}_{1}, \ldots, \mathbf{f}_{b}\right)$. We apply Shearer's lemma to this vector, with $\mathcal{A}$ the collection of sets $\left\{i: y_{i} \in U(x)\right\}$, for $x \in A$. As each $y_{i}$ is in $d$ of these sets, we get

$$
H\left(\mathbf{f}_{B}\right) \leq \frac{1}{d} \sum_{x \in A} H\left(\mathbf{f}_{U(x)}\right) .
$$

Furthermore, noting that $H\left(\mathbf{Y}_{x} \mid \mathbf{f}_{U(x)}\right)=0$, we have

$$
H\left(\mathbf{f}_{U(x)}\right)=H\left(\mathbf{Y}_{x}\right)+H\left(\mathbf{f}_{U(x)} \mid \mathbf{Y}_{x}\right)=\sum_{j=1}^{M} p_{x}(j) \log \left(1 / p_{x}(j)\right)+\sum_{j=1}^{M} p_{x}(j) H\left(\mathbf{f}_{U(x)} \mid \mathbf{Y}_{x}=j\right)
$$


As $|U(x)|=u$ for every $x$, there are exactly $(M-j+1)^{u}-(M-j)^{u}$ values that $\mathbf{f}_{U(x)}$ can take consistent with $\min \mathbf{f}_{U(x)}=\mathbf{Y}_{x}=j$, so

$$
H\left(\mathbf{f}_{U(x)} \mid \mathbf{Y}_{x}=j\right) \leq \log \left[(M-j+1)^{u}-(M-j)^{u}\right]
$$

The term $H\left(\mathbf{f}_{A} \mid \mathbf{f}_{B}\right)$ is at most

$$
\begin{aligned}
& \sum_{x \in A} H\left(\mathbf{f}(x) \mid \mathbf{f}_{B}\right) \leq \sum_{x \in A} H\left(\mathbf{f}(x) \mid \mathbf{Y}_{x}\right), \quad \text { using }(4) \\
= & \sum_{x \in A} \sum_{j=1}^{M} p_{x}(j) H\left(\mathbf{f}(x) \mid \mathbf{Y}_{x}=j\right) \leq \sum_{x \in A} \sum_{j=1}^{M} p_{x}(j) \log j,
\end{aligned}
$$

as, if $\min \mathbf{f}_{U(x)}=\mathbf{Y}_{x}=j$, then $\mathbf{f}(x)$ takes one of the values $1, \ldots, j$.

Combining all these estimates, we have:

$$
\log O_{M}(P) \leq \frac{1}{d} \sum_{x \in A} \sum_{j=1}^{M} p_{x}(j) \log \left(\frac{\left[(M-j+1)^{u}-(M-j)^{u}\right] j^{d}}{p_{x}(j)}\right)
$$

Finally, we apply Jensen's inequality to the sum, and find that

$$
\log O_{M}(P) \leq \frac{1}{d} \sum_{x \in A} \log \left(\sum_{j=1}^{M}\left[(M-j+1)^{u}-(M-j)^{u}\right] j^{d}\right),
$$

so

$$
O_{M}(P) \leq\left(\sum_{j=1}^{M}\left[(M-j+1)^{u}-(M-j)^{u}\right] j^{d}\right)^{a / d}
$$

This is the desired result, as $a u=b d$, so $a / d=b / u=n /(d+u)$.

To deduce Theorem 1.4, we could estimate the sum in Theorem 3.2, but it is easier and more informative to use the result in the form $O_{M}(P) \leq O_{M}\left(K_{d, u}\right)^{n /(d+u)}$.

Proof of Theorem 1.4. For $P$ a poset satisfying the conditions of the theorem, we have, using Theorem 3.2 and Lemma 3.1,

$$
\begin{gathered}
\frac{L(P)}{n !}=\lim _{M \rightarrow \infty} \frac{O_{M}(P)}{M^{n}} \leq \lim _{M \rightarrow \infty} \frac{O_{M}\left(K_{d, u}\right)^{n /(d+u)}}{M^{n}}=\left(\lim _{M \rightarrow \infty} \frac{O_{M}\left(K_{d, u}\right)}{M^{d+u}}\right)^{n /(d+u)} \\
=\left(\frac{L\left(K_{d, u}\right)}{(d+u) !}\right)^{n /(d+u)}=\left(\frac{d ! u !}{(d+u) !}\right)^{n /(d+u)},
\end{gathered}
$$

which is the required result. 
As an example of an application, consider the poset consisting of the middle two levels of the Boolean cube $Q^{t}$, with $t=2 s-1$, so the number of elements is $n=2\left(\begin{array}{c}2 s-1 \\ s\end{array}\right)$. This is a regular bipartite poset with up- and down-degree both equal to $s$, so the number of linear extensions is at most

$$
n !\left(\begin{array}{c}
2 s \\
s
\end{array}\right)^{-n / 2 s} \leq(n / 2) !^{2} \exp ((1+o(1)) n \log s / 4 s) .
$$

We do not know of any other way to obtain this good an estimate.

\section{Proof of Main Theorem}

As in the introduction, let $P$ be a ranked poset with ranks $A_{1}, A_{2}, \ldots, A_{k}$, of sizes $n_{1}, n_{2}$, $\ldots, n_{k}$ respectively, such that every element of $A_{j}$ is below exactly $u_{j}$ elements of $A_{j+1}$ $(j \leq k-1)$, and every element of $A_{j}$ is above exactly $d_{j}$ elements of $A_{j-1}(j \geq 2)$.

While our basic approach will be the same as in the previous section, we have a number of additional difficulties to overcome in this more general case.

Proof of Theorem 1.3. As in the bipartite case, our plan is to bound the number $O_{M}(P)$ of order-preserving maps from $P$ to the chain $C_{M}$, for $M$ suitably larger than $n$, and then to deduce the desired bound on $L(P)$ from it.

Again, let $\mathbf{f}$ be a uniform random variable on the set of all order-preserving maps from $P$ to $C_{M}$, and let $\mathbf{f}_{X}$ denote the restriction of the map $\mathbf{f}$ to the subset $X$. For $x \in A_{i}$ with $i \leq k-1$, set $U(x)=\left\{y \in A_{i+1}: y>x\right\}$ (the set of $u_{i}$ elements covering $x$ ), and let the random variable $\mathbf{B}_{x}$ be $\left\lceil\frac{s}{M} \min \mathbf{f}_{U(x)}\right\rceil$, where $s \leq M$ is fixed and will be specified later. Thus $\mathbf{B}_{x}$ takes an integer value between 1 and $s$ inclusive; we think of $C_{M}$ as divided into blocks of consecutive integers of size $M / s: \mathbf{B}_{x}$ tells us which is the lowest block an element of $U(x)$ is mapped to. Suppose that $x>y$ in $P$, with $\mathbf{B}_{x}=b_{x}$ and $\mathbf{B}_{y}=b_{y}$; then $\mathbf{f}(x) \leq \min \mathbf{f}_{U(x)} \leq \frac{M}{s} b_{x}$, while $\mathbf{f}(x) \geq \min \mathbf{f}_{U(y)}>\frac{M}{s}\left(b_{y}-1\right)$, so $\mathbf{f}(x)$ takes one of at most $\frac{M}{s}\left(b_{x}-b_{y}+1\right)$ values. Therefore,

$$
H\left(\mathbf{f}_{x} \mid \mathbf{B}_{x}=b_{x}, \mathbf{B}_{y}=b_{y}\right) \leq \log \left((M / s)\left(b_{x}-b_{y}+1\right)\right),
$$

which is a crucial bound in what follows.

For $x \in P$ and $b \in[s]$, let $p_{x}(b)$ be the probability that $\mathbf{B}_{x}=b$. For $y<x$ in $P$, and $c \leq b$ in $[s]$, let $p_{y \mid x}(c \mid b)$ be the probability that $\mathbf{B}_{y}=c$ conditioned on $\mathbf{B}_{x}=b$.

We have

$$
\log O_{M}(P)=H(\mathbf{f})=H\left(\mathbf{f}_{A_{k}}\right)+H\left(\mathbf{f}_{A_{k-1}} \mid \mathbf{f}_{A_{k}}\right)+\cdots+H\left(\mathbf{f}_{A_{1}} \mid \mathbf{f}_{A_{2} \cup \cdots \cup A_{k}}\right) .
$$

We claim that, for $j=1, \ldots, k$, the sum of the final $j$ terms above, i.e.,

$$
H\left(\mathbf{f}_{A_{1} \cup \cdots \cup A_{j}} \mid \mathbf{f}_{A_{j+1} \cup \cdots \cup A_{k}}\right),
$$


is bounded above by

$$
\frac{1}{n_{j}} \sum_{x \in A_{j}} \sum_{b_{j}=1}^{s} p_{x}\left(b_{j}\right) \log \left(s^{r_{j}}(M / s)^{n_{1}+\cdots+n_{j}} \max \left[b_{1}^{n_{1}} \prod_{i=2}^{j}\left(b_{i}-b_{i-1}+1\right)^{n_{i}}\right]\right)
$$

where $r_{j}=\sum_{i=2}^{j}\left(n_{i-1} / d_{i}\right)$ (so $r_{k}=r$ ) and the maximum is taken over all $\left(b_{1}, \ldots, b_{j-1}\right)$ with $1 \leq b_{1} \leq b_{2} \leq \cdots \leq b_{j}$ : it is well-known and easy to check (using Lagrange multipliers) that this maximum is attained by taking each $b_{i}-b_{i-1}+1$ proportional to $n_{i}$ (where $b_{0}:=1$ ).

We prove the above claim by induction on $j$. For $j=1$, we argue that:

$$
\begin{gathered}
H\left(\mathbf{f}_{A_{1}} \mid \mathbf{f}_{A_{2} \cup \cdots \cup A_{k}}\right) \leq \sum_{x \in A_{1}} H\left(\mathbf{f}(x) \mid \mathbf{f}_{A_{2} \cup \cdots \cup A_{k}}\right) \leq \sum_{x \in A_{1}} H\left(\mathbf{f}(x) \mid \mathbf{B}_{x}\right) \\
=\sum_{x \in A_{1}} \sum_{b_{1}=1}^{s} p_{x}\left(b_{1}\right) H\left(\mathbf{f}_{x} \mid \mathbf{B}_{x}=b_{1}\right) \leq \sum_{x \in A_{1}} \sum_{b_{1}=1}^{s} p_{x}\left(b_{1}\right) \log \left[(M / s) b_{1}\right] \\
=\frac{1}{n_{1}} \sum_{x \in A_{1}} \sum_{b_{1}=1}^{s} p_{x}\left(b_{1}\right) \log \left[(M / s)^{n_{1}} b_{1}^{n_{1}}\right]
\end{gathered}
$$

which is as claimed. (Note that $r_{1}=0$.)

For the induction step, we assume the result for $j-1$, and consider the new term $H\left(\mathbf{f}_{A_{j}} \mid \mathbf{f}_{A_{j+1} \cup \ldots \cup A_{k}}\right)$. This is at most

$$
\frac{1}{d_{j}} \sum_{y \in A_{j-1}} H\left(\mathbf{f}_{U(y)} \mid \mathbf{f}_{A_{j+1} \cup \cdots \cup A_{k}}\right), \quad \text { by Shearer's lemma }
$$

$\leq \frac{1}{d_{j}} \sum_{y \in A_{j-1}}\left(H\left(\mathbf{B}_{y} \mid \mathbf{f}_{A_{j+1} \cup \cdots \cup A_{k}}\right)+H\left(\mathbf{f}_{U(y)} \mid \mathbf{B}_{y}, \mathbf{f}_{A_{j+1} \cup \cdots \cup A_{k}}\right)\right)$, using the conditional version of (5)

$$
\begin{aligned}
& \leq \frac{1}{d_{j} u_{j-1}} \sum_{y \in A_{j-1}} \sum_{x \in U(y)} H\left(\mathbf{B}_{y} \mid \mathbf{B}_{x}\right)+\frac{1}{d_{j}} \sum_{y \in A_{j-1}} \sum_{x \in U(y)} H\left(\mathbf{f}_{x} \mid \mathbf{B}_{y}, \mathbf{B}_{x}\right), \text { using (4) } \\
& \quad=\frac{1}{d_{j} u_{j-1}} \sum_{y \in A_{j-1}} \sum_{x \in U(y)} \sum_{b_{j}=1}^{s} p_{x}\left(b_{j}\right) \sum_{b_{j-1}=1}^{b_{j}} p_{y \mid x}\left(b_{j-1} \mid b_{j}\right) \log \left(1 / p_{y \mid x}\left(b_{j-1} \mid b_{j}\right)\right)+ \\
& \quad+\frac{1}{d_{j}} \sum_{y \in A_{j-1}} \sum_{x \in U(y)} \sum_{b_{j}=1}^{s} p_{x}\left(b_{j}\right) \sum_{b_{j-1}=1}^{b_{j}} p_{y \mid x}\left(b_{j-1} \mid b_{j}\right) H\left(\mathbf{f}_{x} \mid \mathbf{B}_{y}=b_{j-1}, \mathbf{B}_{x}=b_{j}\right) \\
& \leq \frac{1}{d_{j} u_{j-1}} \sum_{y \in A_{j-1}} \sum_{x \in U(y)} \sum_{b_{j}=1}^{s} p_{x}\left(b_{j}\right) \sum_{b_{j-1}=1}^{b_{j}} p_{y \mid x}\left(b_{j-1} \mid b_{j}\right) \log \left(\frac{(M / s)^{u_{j-1}}\left(b_{j}-b_{j-1}+1\right)^{u_{j-1}}}{p_{y \mid x}\left(b_{j-1} \mid b_{j}\right)}\right)
\end{aligned}
$$

using (7). 
By the induction hypothesis, the sum of the final $j-1$ terms is at most

$$
\frac{1}{n_{j-1}} \sum_{y \in A_{j-1}} \sum_{b_{j-1}=1}^{s} p_{y}\left(b_{j-1}\right) \log X\left(b_{j-1}\right)
$$

where

$$
X\left(b_{j-1}\right)=s^{r_{j-1}}(M / s)^{n_{1}+\cdots+n_{j-1}} \max \left[b_{1}^{n_{1}} \cdots\left(b_{j-1}-b_{j-2}+1\right)^{n_{j-1}}\right],
$$

and this sum can be rewritten as

$$
\frac{1}{n_{j-1} u_{j-1}} \sum_{y \in A_{j-1}} \sum_{x \in U(y)} \sum_{b_{j-1}=1}^{s} \sum_{b_{j}=b_{j-1}}^{s} p_{x}\left(b_{j}\right) p_{y \mid x}\left(b_{j-1} \mid b_{j}\right) \log X\left(b_{j-1}\right) .
$$

Therefore the sum of all the relevant terms is at most

$$
\begin{aligned}
& \frac{1}{d_{j} u_{j-1}} \sum_{y \in A_{j-1}} \sum_{x \in U(y)} \sum_{b_{j}=1}^{s} p_{x}\left(b_{j}\right) \sum_{b_{j-1}=1}^{b_{j}} p_{y \mid x}\left(b_{j-1} \mid b_{j}\right) \log \left(\frac{(M / s)^{u_{j-1}}\left(b_{j}-b_{j-1}+1\right)^{u_{j-1}}\left(X\left(b_{j-1}\right)\right)^{d_{j} / n_{j-1}}}{p_{y \mid x}\left(b_{j-1} \mid b_{j}\right)}\right) \\
& \leq \frac{1}{d_{j} u_{j-1}} \sum_{x \in A_{j}} \sum_{\substack{y \in A_{j-1} \\
y<x}} \sum_{b_{j}=1}^{s} p_{x}\left(b_{j}\right) \log \left(\sum_{b_{j-1}=1}^{b_{j}}(M / s)^{u_{j-1}}\left(b_{j}-b_{j-1}+1\right)^{u_{j-1}}\left(X\left(b_{j-1}\right)\right)^{d_{j} / n_{j-1}}\right),
\end{aligned}
$$

by Jensen's inequality, and the above is in turn at most

$$
\leq \frac{1}{u_{j-1}} \sum_{x \in A_{j}} \sum_{b_{j}=1}^{s} p_{x}\left(b_{j}\right) \log \left(s(M / s)^{u_{j-1}} \max _{b_{j-1}}\left[\left(b_{j}-b_{j-1}+1\right)^{u_{j-1}} X\left(b_{j-1}\right)^{d_{j} / n_{j-1}}\right]\right)
$$

(now we use that $\left.1 / u_{j-1}=\left(n_{j-1} / d_{j}\right)\left(1 / n_{j}\right)\right)$

$$
\begin{aligned}
& =\frac{1}{n_{j}} \sum_{x \in A_{j}} \sum_{b_{j}=1}^{s} p_{x}\left(b_{j}\right) \log \left(s^{n_{j-1} / d_{j}}(M / s)^{n_{j}} \max _{b_{j-1}}\left[\left(b_{j}-b_{j-1}+1\right)^{n_{j}} X\left(b_{j-1}\right)\right]\right) \\
& =\frac{1}{n_{j}} \sum_{x \in A_{j}} \sum_{b_{j}=1}^{s} p_{x}\left(b_{j}\right) \log \left(s^{r_{j}}(M / s)^{n_{1}+\cdots+n_{j}} \max \left[b_{1}^{n_{1}} \prod_{i=2}^{j}\left(b_{i}-b_{i-1}+1\right)^{n_{i}}\right]\right),
\end{aligned}
$$

which completes the induction step.

Carrying it through to the end, we can treat $\mathbf{B}_{x}$ as identically equal to $s$ for $x$ in the top class $A_{k}$, and we emerge with

$O_{M}(P) \leq s^{r}(M / s)^{n_{1}+\cdots+n_{k}} \max b_{1}^{n_{1}}\left(b_{2}-b_{1}+1\right)^{n_{2}} \cdots\left(b_{k-1}-b_{k-2}+1\right)^{n_{k-1}}\left(s-b_{k-1}+1\right)^{n_{k}}$.

Now we do the maximization and, setting $n=n_{1}+\cdots+n_{k}$, we get:

$$
\begin{gathered}
O_{M}(P) \leq s^{r}(M / s)^{n}\left(\frac{n_{1}}{n}(s+k-1)\right)^{n_{1}} \cdots\left(\frac{n_{k}}{n}(s+k-1)\right)^{n_{k}} \\
=s^{r}(M / s)^{n}(s+k-1)^{n} \frac{n_{1}^{n_{1}} \cdots n_{k}^{n_{k}}}{n^{n}} .
\end{gathered}
$$


For any $M$, we certainly have $O_{M}(P) \geq L(P)\left(\begin{array}{c}M \\ n\end{array}\right)$. Therefore

$$
\begin{aligned}
& L(P) \leq s^{r} M^{n}\left(1+\frac{k-1}{s}\right)^{n} \frac{n_{1}^{n_{1}} \cdots n_{k}^{n_{k}}}{n^{n}} \frac{n !}{(M-n)^{n}} \\
& \leq s^{r}\left(1+\frac{n}{M-n}\right)^{n}\left(1+\frac{k-1}{s}\right)^{n} n_{1} ! \cdots n_{k} !
\end{aligned}
$$

Taking the limit as $M \rightarrow \infty$ now yields, for any choice of $s$ :

$$
L(P) \leq s^{r} e^{(k-1) n / s} n_{1} ! \cdots n_{k} ! .
$$

Finally we optimize this bound by setting $s=\lceil(k-1) n / r\rceil$; as $r \leq n$ and $k-1 \geq 1$, we have $(k-1) n / r \leq s \leq 2(k-1) n / r$. Hence

$$
L(P) \leq\left(\frac{2(k-1) n}{r}\right)^{r} e^{r} n_{1} ! \cdots n_{k} !
$$

proving the theorem.

Proof of Theorem 1.1. Recall that $r=\sum_{j=2}^{k}\left(n_{j-1} / d_{j}\right)$. For the Boolean cube $P=Q^{t}$, we have $n=2^{t}, k=t+1, n_{j}=\left(\begin{array}{c}t \\ j-1\end{array}\right)$ and $d_{j}=j-1$, so

$$
r=\sum_{i=0}^{t-1}\left(\begin{array}{l}
t \\
i
\end{array}\right) \frac{1}{i+1}=\frac{1}{t+1}\left(2^{t+1}-2\right)<2 n / t .
$$

Therefore, by Theorem 1.3, we have, for $t \geq 3$,

$$
L\left(Q^{t}\right) \leq\left(e t^{2}\right)^{2 n / t} \prod_{j=0}^{t}\left(\begin{array}{l}
t \\
j
\end{array}\right) ! \leq e^{6 n \ln t / t} \prod_{j=0}^{t}\left(\begin{array}{l}
t \\
j
\end{array}\right) !
$$

Of course, we have $L\left(Q^{t}\right)=\prod\left(\begin{array}{l}t \\ j\end{array}\right)$ ! for $t<3$, so the result follows.

\section{$5 \quad$ LYM posets}

Let $P$ be a ranked poset on $[n]$ with ranks $A_{1}, A_{2}, \ldots, A_{k}$ of sizes $n_{1}, n_{2}, \ldots, n_{k}$ respectively. The weight $w(x)$ of an element $x \in A_{j}$ is defined to be $1 / n_{j}$. The $L Y M$ condition is that, for any antichain $A$ of $P$, the sum $w(A)$ of the weights of elements of $A$ is at most 1 . (Note that equality is achieved for each of the ranks $A_{j}$.) 
Theorem 5.1 Let $P$ be a ranked poset satisfying the LYM condition, with rank sizes $n_{1}, \ldots, n_{k}$. Then $L(P) \leq \prod_{j=1}^{k} n_{j}^{n_{j}}$.

The well-known LYM theorem states that the Boolean lattice $Q^{t}$ satisfies the LYM condition (see for instance Bollobás [1]), so Theorem 5.1 extends the result of Sha and Kleitman [14], as well as that of Shastri [15], who proved it under the additional assumption that $\left(n_{i}\right)$ is a symmetric unimodal sequence.

Theorem 5.1 is a special case of the following result from Kahn and Kim [11].

Theorem 5.2 Let $P$ be any n-element poset, and let $w(x)$ be any non-negative function on $P$ satisfying $w(A)=\sum_{x \in A} w(x) \leq 1$ for all antichains $A$ of $P$. Then $e(P) \leq$ $\prod_{x \in P} 1 / w(x)$.

Theorem 5.1 follows immediately from Theorem 5.2, as the function given by $w(x)=$ $1 / n_{j}$ for $x \in A_{j}$ satisfies the hypotheses, so that each of the $n_{j}$ elements of $A_{j}$ contributes a factor of $n_{j}$ to the product $\prod_{x \in P} 1 / w(x)$.

Kahn and Kim's proof of Theorem 5.2 uses polyhedral methods, and is based on a theorem of Stanley. (They go on to use these techniques to prove important results on comparison sorting algorithms.) A research report [3] of the first author contains further information on this topic, and also gives an alternative proof of Theorem 5.2, which we include here as it is very short and natural, and may be of some interest.

Proof. Consider the following random procedure for building a linear extension of $P$ from the bottom up. At each stage, from the set of available elements, choose $x$ as the next element with probability proportional to $w(x)$.

Now consider any single linear extension $\prec$ of $P$, say $x_{1} \prec x_{2} \prec \cdots \prec x_{n}$. The probability that our random procedure results in $\prec$ is exactly $\prod_{i=1}^{n} w\left(x_{i}\right) / w\left(B_{i}\right)$, where $B_{i}$ is the set of elements minimal among $\left\{x_{i}, \ldots, x_{n}\right\}$. Since the $B_{i}$ are antichains, each $w\left(B_{i}\right)$ is at most 1 , and the probability of $\prec$ is at least $\prod_{i=1}^{n} w\left(x_{i}\right)$. This is independent of the particular linear extension $\prec$, so we conclude that the total number of possible linear extensions is at most $1 / \prod w\left(x_{i}\right)$, as required.

The disjoint union $P$ of $n / k$ chains, each of $k$ elements, is an LYM poset, and

$$
L(P)=n ! / k !^{n / k}=\left(\frac{n}{k}(1-o(1))\right)^{n}
$$

in close agreement with the upper bound in Theorem 5.1. What this means is that Theorem 5.1 gives (in some sense) close to the best possible result for LYM posets in general, so that information on the degrees was essential for our improved upper bound in the case of the Boolean lattice. 
Acknowledgments. We thank the Institute for Mathematics and Applications (IMA) for funding the summer school in Combinatorics and Applications at Georgia Tech. during July 2003. This was pivotal to our collaboration. We also thank Tom Trotter and the staff of the School of Mathematics (Georgia Tech.) for organizing and hosting the summer school. Finally, we thank the anonymous referee for a very thorough checking of our manuscript.

\section{References}

[1] B. Bollobás, Combinatorics, Cambridge University Press 1986.

[2] B. Bollobás and G.R. Brightwell, The number of k-SAT functions, Random Structures and Algorithms 22 (2003) 227-247.

[3] G.R. Brightwell, The number of linear extensions of ranked posets, LSE-CDAM Research Report LSE-CDAM-2003-18 (2003).

[4] F.R.K. Chung, P. Frankl, R. Graham and J.B. Shearer, Some intersection theorems for ordered sets and graphs, J. Combin. Theory Ser. A. 48 (1986), 23-37.

[5] T. Cover and J. Thomas, Elements of Information Theory, John Wiley, New York (1991).

[6] I. Csiszár and J. Körner, Information Theory: Coding Theorems for discrete memoryless systems, Academic Press, New York (1981).

[7] P.C. Fishburn and W.T. Trotter, Linear extensions of semiorders: a maximization problem, Discrete Math. 103 (1992) 25-40.

[8] D. Galvin and P. Tetali, On weighted graph homomorphisms, Special DIMACS-AMS volume on Graph Homomorphisms and Statistical Physics Models, to appear.

[9] J. Kahn, An entropy approach to the hard-core model on bipartite graphs, Combin. Prob. Comp. 10 (2001), 219-237.

[10] J. Kahn, Entropy, independent sets and antichains: a new approach to Dedekind's problem, Proc. Amer. Math. Soc. 130 (2002), 371-378.

[11] J. Kahn and J.H. Kim, Entropy and sorting, J. Comput. System Sci. 51 (1995) 390-399

[12] R.J. McEliece, The Theory of Information and Coding, Addison-Wesley, London, 1977.

[13] J. Radhakrishnan, Entropy and Counting, Preprint of a survey article (November 2001).

[14] J. Sha and D. J. Kleitman, The number of linear extensions of subset ordering, Discrete Math., 63 (1987), 271-279. 
[15] A. Shastri, Number of linear extensions of symmetric KLYM posets, Util. Math. 53 (1998) 25-35.

[16] L.A. Shepp, The FKG inequality and some monotonicity properties of partial orders, SIAM J. Algebraic Discrete Methods 1 (1980) 295-299.

[17] L. Shepp, The $X Y Z$ conjecture and the FKG inequality, Ann. Probab. 10 (1982) 824-827. 